\title{
Test Function Spaces for Direct Product Representations of the Canonical Commutation Relations
}

\author{
L. Streit \\ ETH Zürich, Seminar für Theoretische Physik
}

Received July 1, 1966

\begin{abstract}
It is shown how the test function spaces for the field operator and its canonical conjugate are determined by a given irreducible direct product representation of the canonical commutation relations. An explicit characterization of the admissible test functions (so that the smeared out field operators are selfadjoint) is given in terms of any one product state of the representation space.
\end{abstract}

\section{§1. Introduction}

Regarding myriotic representations of the canonical commutation relations there exists by now - in addition to general statements [1] a variety of field theoretical models which illustrate their significance.

Examples are the quantum theory of optical coherence [2] (in the general case of infinite average photon number) and methods for the elimination of infrared divergences [3], [4], the infinite free Bose gas of non-zero density [5] and (in the Fermion case) the HAAG solution [6] of the BCS-model.

The mathematical properties of a large class of inequivalent representations have recently been studied by KLAUDER et al. [7]. These "direct product" representations are generated by defining the Weyl operators $e^{-i \pi(g)}, e^{i \varphi(f)}$ roughly as follows:

Let

$$
\begin{aligned}
e^{i \varphi(f)} & =e^{i \Sigma Q_{\nu} s_{\nu}}=W(\underline{s}) \\
e^{-i \pi(g)} & =e^{-i \Sigma P_{\nu} t_{\nu}}=V(\underline{t})
\end{aligned}
$$

where $f(x)=\Sigma s_{\nu} h_{v}(x)=(\underline{s}, \underline{h}), g(x)=(\underline{t}, \underline{h}), \underline{h}$ a complete orthonormal set of functions and where the $P_{\nu}, Q_{\nu}$ act as canonical conjugate operators on the $v^{\text {th }}$ factor of an infinite direct product

$$
H=\prod_{v=1}^{\infty} \otimes H_{\nu}
$$

of "oscillator" Hilbert spaces $H_{v}$.

$H$ is non-separable [8], the representations so obtained are highly reducible, and a decomposition into irreducible representations can be given. 
KLAUder, McKenna and Woods [7] have proved a criterion for the inequivalence of such irreducible representations which we will derive anew making strong use of the theory of von Neumann algebras.

The topological properties of the latter allow to avoid an a priori definition of the test function spaces $\{f\}$ and $\{g\}$. In fact the class of sequences $\underline{s}, \underline{t}$ which correspond to unitary operators depends strongly on the given irreducible representation; one may show [3] that

a) every representation allows for some infinite $\underline{s}, \underline{t}$

b) for any given infinite sequence $\underline{s}$ or $\underline{t}$ there exist irreducible representations such that the restrictions of $W(\underline{s})$ or $V(\underline{t})$ are not unitary i.e. such that $f=(\underline{s}, \underline{h})$ or $g=(\underline{t}, \underline{h})$ are no good testfunctions.

In the following we shall derive criteria for $\underline{\underline{s}} \underline{t}$ that are necessary and sufficient for $f$ and $g$ to be good test functions in the above sense.

$\S 2$ will contain a collection of definitions, in $\S 3$ we shall give our proof of the KLaUder, McKenna and Woods theorem, $\S 4$ will serve to characterize the test function spaces.

\section{§2. Definitions and other preliminaries}

A. Concerning infinite direct product spaces and following v. NeUMANN [8]: The infinite direct product of Hilbert spaces $H_{\nu}$

$$
H=\prod_{v} \otimes H_{v}
$$

is generated by linear combinations of " $c_{0}$-vectors" $\chi$

$$
\chi=\prod_{v} \otimes \chi_{v} \quad \chi_{v} \in H_{v} \quad \sum_{v}\left|\left\|\chi_{v}\right\|-1\right|<\infty
$$

with the scalar product

$$
(\chi, \psi)=\prod_{\nu}\left(\chi_{v}, \psi_{v}\right)
$$

Two $c_{0}$-vectors $\chi, \psi$ are called equivalent if

$$
\sum_{\nu}\left|\left(\chi_{v}, \psi_{v}\right)-1\right|<\infty \quad(\chi \sim \psi)
$$

and weakly equivalent if

$$
\sum_{\nu}||\left(\chi_{\nu}, \psi_{\nu}\right)|-1|<\infty \quad(\chi \underset{w}{\sim} \psi) .
$$

In the latter case the weak equivalence class $c_{w}(\chi)$ contains the whole equivalence class $c(\psi)$ :

$$
c(\chi) \underset{w}{\sim} c(\psi) .
$$

The $c_{0}$-vectors of a given equivalence class $c$ span the incomplete direct product space (IDPS) denoted by $\Pi \stackrel{c}{\oplus} H_{\nu}$. 
The algebra of all bounded linear operators in $H_{v}$

$$
B\left(H_{\nu}\right)=\left\{A_{\nu}\right\}
$$

is extended to an algebra $\bar{B}\left(H_{v}\right)$ of operators

$$
\bar{A}_{v}=\mathbf{1}_{1} \otimes \cdots \otimes \mathbf{1}_{v-1} \otimes A_{\nu} \otimes \mathbf{1}_{v+1} \otimes \cdots \in B(H)
$$

and generates the von Neumann algebra of extended operators

$$
B^{\#}=\left(\bigcup_{v} \bar{B}\left(H_{v}\right)\right)^{\prime \prime} \subset B(H) .
$$

Finally we denote the restriction of an operator $A \in B^{\#}$ to $\Pi^{c} \otimes H_{\nu}$ by $A^{c}$.

B. Concerning the canonical commutation relations ( $\left.{ }^{\nu} \mathrm{CR}\right)$ : The $H_{v}$ are identified with "oscillator" Hilbert spaces $H_{v}=L_{2}\left(R_{1}\right)$ with $q_{v}=\xi$

$$
\begin{aligned}
p_{\nu} & =-i \frac{\partial}{\partial \xi} \\
V_{\nu}(t) & =e^{-i p_{\nu} t} \in B\left(H_{v}\right) \\
W_{\nu}(s) & =e^{i q_{\nu} s} \in B\left(H_{v}\right) \\
M_{\nu} & =\left\{V_{\nu}(t), W_{\nu}(s) \mid-\infty<s, t<+\infty\right\} \\
\widetilde{V}(\underline{t}) & =\prod_{\nu} \otimes e^{-i p_{\nu} t_{\nu}} \in B(H) \\
\widetilde{W}(\underline{s}) & =\prod_{\nu} \otimes e^{i q_{\nu} s_{v}} \in B(H)
\end{aligned}
$$

are defined by the equations

$$
\begin{aligned}
& \widetilde{V}(\underline{t}) \chi=\prod_{\nu} \otimes e^{-i p_{v} t_{v}} \chi_{v} \\
& \widetilde{W}(\underline{s}) \chi=\prod_{\nu} \otimes e^{i q_{v} s_{v}} \chi_{v} .
\end{aligned}
$$

We shall write $\widetilde{V}^{c}(\underline{t})=V(\underline{t}), \widetilde{W}^{c}(\underline{s})=W(\underline{s})$ to simplify the notation and shall denote rational $\underline{s}$ and $\underline{t}$ (sequences with $s_{v}, t_{v}=0$ for almost all $v$ ) by $\underline{s}^{(r)}$ and $\underline{t}^{(r)}$.

\section{\$3. The irreducible direct product representations}

For the $\widetilde{V}\left(\underline{t}^{(r)}\right), \widetilde{W}\left(\underline{s}^{(r)}\right)$ one verifies directly that they are unitary operators in $B(H)$ which obey the CCR:

$$
\begin{aligned}
\widetilde{V}\left(\underline{t}_{1}^{(r)}\right) \widetilde{V}\left(\underline{t}_{2}^{(r)}\right) & =\widetilde{V}\left(\underline{t}_{1}^{(r)}+\underline{t}_{2}^{(r)}\right) \\
\widetilde{W}\left(\underline{s}_{1}^{(r)}\right) \widetilde{W}\left(\underline{s}_{2}^{(r)}\right) & =\widetilde{W}\left(\underline{s}_{1}^{(r)}+\underline{s}_{2}^{(r)}\right) \\
\widetilde{V}(0) & =\widetilde{W}(0)=1 \\
\widetilde{V}\left(\underline{t}^{(r)}\right) \widetilde{W}\left(\underline{s}^{(r)}\right) & =e^{-i \Sigma s_{\nu} t_{\nu}} \widetilde{W}\left(\underline{s}^{(r)}\right) \widetilde{V}\left(\underline{t}^{(r)}\right) .
\end{aligned}
$$

Note that $\Sigma s_{v} t_{v}$ is a finite sum for rational $\underline{s}, \underline{t}$, so that there is no convergence problem. Furthermore

$$
\chi \sim \widetilde{V}\left(\underline{t}^{(r)}\right) \chi \sim \widetilde{W}\left(\underline{s}^{(r)}\right) \chi
$$


since such $\widetilde{V}, \widetilde{W}$ are equal to $\mathbf{1}_{\nu}$ on almost every $H_{v}$. This suggests already that the decomposition of $H$ into incomplete direct product spaces will reduce the representation. To make the argument rigorous we use the following lemmata:

Lemma 1 [9]. $M_{v}^{\prime \prime} \equiv\left\{V_{v}(s), W_{v}(t) \mid-\infty<s, t<\infty\right\}^{\prime \prime}=B\left(H_{v}\right)$.

Lemma 2. Let $K$ be a Hilbert space, $I$ an index set, $M_{v} \leqq B(K) \forall v \in I$

$$
\Rightarrow\left(\bigcup_{\nu \in I} M_{v}\right)^{\prime \prime}=\left(\bigcup_{\nu \in I} M_{v}^{\prime \prime}\right)^{\prime \prime} .
$$

(For a proof cf. e.g. [10].)

From these one immediately gets

Lemma 3. $B^{\#}=\left\{\widetilde{V}\left(\underline{t}^{(r)}\right), \widetilde{W}\left(\underline{s}^{(r)}\right) \mid \text { all } \underline{s}^{(r)}, \underline{t}^{(r)}\right\}^{\prime \prime}$.

As a consequence of a theorem given by v. Neumann [8] we have the

Lemma 4. Let $A \in B\left(\underset{v}{\prod_{\nu}^{c}} H_{v}\right)$

$$
\Rightarrow \exists Q \in B^{\#} \quad \text { with } \quad Q^{c}=A \quad Q^{c^{\prime}}=0 \forall c_{w}^{\prime} \underset{w}{c} .
$$

Lemmata 3 and 4 serve to show the irreducibility of $\left\{\widetilde{V}\left(\underline{t}^{(r)}\right), \widetilde{W}\left(\underline{s}^{(r)}\right)\right\}$ restricted to $\Pi^{c} \otimes H_{v}$, since

$$
\left(\left\{\widetilde{V}\left(t^{(r)}\right), \widetilde{W}\left(\underline{\tilde{s}}^{(r)}\right)\right\}^{\prime \prime}\right)^{c}=\left(B^{\#}\right)^{c}=B\left(\prod_{\nu}^{c} H_{v}\right)
$$

and, since restriction (c) and completion $\left(^{(\prime)}\right.$ may be permuted [11], the restricted rational Weyl operators generate the full algebra $B\left(\prod_{\nu}^{c} H_{\nu}\right)$ qed. - Furthermore we note that they are cyclic [12]:

$$
\left\{V\left(\underline{t}^{(r)}\right), W\left(\underline{s}^{(r)}\right)\right\}^{\prime}=\{\alpha \mathbf{1}\} \text { in } \prod_{\nu}^{c} \otimes H_{\nu} .
$$

To decide which of the so obtained irreducible representations are inequivalent there is the

Theorem (KLauder, McKenna and Woods, ref. [7]). The representations $\left\{\widetilde{V}\left(\underline{t}^{(r)}\right), \widetilde{W}\left(\underline{s}^{(r)}\right)\right\}^{c_{i}}, i=1,2$, are equivalent if and only if $c_{\mathbf{1}} \underset{w}{\tilde{w}} c_{\mathbf{2}}$.

Proof. (We shall skip the straightforward "if"-part and give only our version of the "only if" part.)

Equivalence of the representations implies the existence of a unitary transform

with

$$
T \in B\left(\prod_{\nu}^{c_{1}} H_{v}, \prod_{\nu}^{c_{2}} H_{v}\right)
$$

$$
\begin{aligned}
T \widetilde{V}^{c_{1}}\left(\underline{t}^{(r)}\right) T^{+} & =\widetilde{V}^{c_{2}}\left(\underline{t}^{(r)}\right) \\
T \widetilde{W}^{c_{1}}\left(\underline{s}^{(r)}\right) T^{+} & =\widetilde{W}^{c_{2}}\left(\underline{s}^{(r)}\right)
\end{aligned}
$$

i.e.

$$
T Q^{c_{1}} T^{+}=Q^{c_{2}} \quad \forall Q \in\left\{\widetilde{V}\left(\underline{t}^{(r)}\right), \widetilde{W}\left(\underline{s}^{(r)}\right)\right\}
$$


and consequently

$$
\forall Q \in\left\{\widetilde{V}\left(\underline{t}^{(r)}\right), \widetilde{W}\left(\underline{s}^{(r)}\right)\right\}^{\prime \prime}=B^{\#} \text { (lemma 3) . }
$$

But from lemma 4 we know that there are $Q \in B^{\#}$ with $Q^{c_{1}}=1, Q^{c_{2}}=0$ if only $c_{1} \underset{w}{\sim} c_{2}$, which contradicts unitary equivalence qed.

\section{$\$ 4$. The test function spaces}

The object of this paragraph will be to find the sets $\sigma, \tau$ of all (infinite, real) sequences $\underline{s}, \underline{t}$ such that $V(\lambda \underline{t}), W(\lambda \underline{s})$ are unitary, obey the CCR and are weakly continuous in $\lambda$ so that through Stone's theorem [13] they generate self-adjoint field operators

$$
\begin{array}{rr}
\varphi(f)=\lim _{\lambda \rightarrow 0}(i \lambda)^{-1}(W(\lambda \underline{s})-1) \quad f=(\underline{s}, \underline{h}) \\
\pi(g)=\lim _{\lambda \rightarrow 0}(-i \lambda)^{-1}(V(\lambda \underline{t})-1) \quad g=(\underline{t}, \underline{h}) .
\end{array}
$$

As a first step we shall verify that unitarity alone already characterizes $\sigma$ and $\tau$.

\section{Definition.}

$$
\begin{aligned}
& \sigma \equiv\left\{\underline{s} \mid W(\lambda \underline{s}) \in B\left(\prod_{\nu}^{c} \otimes H_{v}\right) \text { unitary } \forall \text { real } \lambda\right\} . \\
& \tau \equiv\left\{\underline{t} \mid V(\lambda \underline{t}) \in B\left(\Pi_{v}^{c} \otimes H_{v}\right) \quad \text { unitary } \forall \text { real } \lambda\right\} .
\end{aligned}
$$

(One will expect $\sigma$ and $\tau$ to depend on the equivalence class $c$; i.e. on the given irreducible representation of the CCR.)

With infinite sequences $\underline{a}$ we associate rational sequences $\underline{a}_{n}$ the first $n$ elements of which coincide with those of $\underline{a}$ whereas the rest are zero:

$$
a_{n, v}=\left\{\begin{array}{l}
a_{v} v \leqq n \\
0 \quad v>n
\end{array}\right.
$$

For simplicity we shall not use an upper index $(r)$ here.

The following lemma relates the infinite sequences $\underline{s}, \underline{t}$ to rational ones and will thus enable us to see that continuity and CCR for $W(\lambda \underline{s}), V(\lambda \underline{t})$ extend to all $\underline{s} \in \sigma, \underline{t} \in \tau$.

Then

Lemma 5. Let $\underline{s} \in \sigma, \underline{t} \in \tau$

$$
\begin{gathered}
\mathrm{s}-\lim _{n} W\left(\underline{s}_{n}\right)=W(\underline{s}) \\
\mathrm{s}-\lim _{n} V\left(\underline{t}_{n}\right)=V(\underline{t}) .
\end{gathered}
$$

Proof. For any two $c_{0}$-vectors $\chi, \psi \in \Pi \stackrel{c}{\otimes} H_{\nu}$

$$
\begin{aligned}
\lim _{n}\left(\chi, W\left(\underline{s}_{n}\right) \psi\right) & =\lim _{n} \prod_{\nu=1}^{n}\left(\chi_{v}, W\left(s_{v}\right) \psi_{\nu}\right) \prod_{\nu=n+1}^{\infty}\left(\chi_{v}, \psi_{v}\right) \\
& =\lim _{n} \prod_{\nu=1}^{n}\left(\chi_{\nu}, W\left(s_{v}\right) \psi_{v}\right) \\
& =(\chi, W(\underline{s}) \psi)
\end{aligned}
$$


the second equality following from the equivalence of $\chi$ and $\psi$. Because it is sesquilinear, the resulting relation

$$
\left(\chi, W\left(\underline{s}_{n}\right) \psi\right) \rightarrow(\chi, W(\underline{s}) \psi)
$$

is true if only $\chi$ and $\psi$ are elements of the set $M$ of finite linear combinations of $c_{0}$-vectors; $M$ is dense in $\Pi^{c} \otimes H_{v}$. Consequently

$$
\begin{aligned}
&\left\|\left(W\left(\underline{s}_{n}\right)-W(\underline{s})\right) \psi\right\|^{2}=2\|\psi\|^{2}-2 \operatorname{Re}\left(W(\underline{s}) \psi, W\left(\underline{s}_{n}\right) \psi\right) \rightarrow 0 \\
& \text { or } \quad W\left(\underline{s}_{n}\right) \psi \rightarrow W(\underline{s}) \psi
\end{aligned}
$$

for all $\psi \in M$ (note that $\chi \equiv W \psi$ is in $M$ for all $\psi$ from $M$ ), but for bounded operators strong vector convergence

$$
W_{n} \psi \rightarrow W \psi
$$

for all $\psi$ from a dense linear manifold entails strong operator convergence [14]

or in our case

$$
W_{n} \rightarrow W
$$

and analogously

$$
\operatorname{s-lim} W\left(\underline{s}_{n}\right)=W(\underline{s})
$$

$$
\text { s- } \lim _{n} V\left(\underline{t}_{n}\right)=V(\underline{t}) \quad \text { qed. }
$$

Lemma 6. $\forall \underline{s} \in \sigma, \underline{t} \in \tau W(\lambda \underline{s})$ and $V(\lambda \underline{t})$ obey the CCR and are continuous in $\lambda$.

Proof. $W(\underline{s}) W\left(\underline{s}^{\prime}\right)=W\left(\underline{s+s^{\prime}}\right) \quad s, s^{\prime} \in \sigma \quad \underline{s+s^{\prime}} \equiv\left\{s_{\nu}+s_{v}^{\prime}\right\}$ and the analogue for $V(\underline{t})$ follow directly from the definitions of $V$ and $W$.

As the operator product is continuous in the sense of strong convergence (although not in the sense of strong topology) [15], lemma 5 guarantees the existence of

$$
\lim _{n} e^{i \lambda \sum_{\nu=1}^{n} s_{\nu} t_{\nu}}=\lim _{n \rightarrow \infty} W\left(\lambda \underline{s}_{n}\right) V\left(\underline{t}_{n}\right) W\left(-\lambda \underline{s}_{n}\right) V\left(-\underline{t}_{n}\right) .
$$

Noting that the above relation holds for all real $\lambda$ we may write

$$
\lim _{n} e^{i \lambda} \sum_{\nu=1}^{n} s_{\nu} t_{\nu}=e^{i \lambda \sum_{\nu=0}^{\infty} s_{\nu} t_{\nu}} \quad \text { with } \quad \sum_{\nu=1}^{\infty} s_{\nu} t_{\nu}<\infty
$$

thus completing the proof of the CCR.

Continuity of $W(\lambda \underline{s})$ is implied by the facts that $W(\lambda \underline{s})$ is unitary, that its matrix elements

$$
(\varphi, W(\lambda \underline{s}) \psi)=\lim _{n}\left(\varphi, W\left(\lambda \underline{s}_{n}\right) \psi\right)
$$

are measurable functions in $\lambda$ and by the separability [7] of the IDPS [16]. Again the analogue is true for $V(\lambda \underline{t})$. 
With the result of lemma 6 in mind the task of finding the test function spaces for $\varphi(x)$ and $\pi(x)$ reduces to that of making the dependence of $\sigma$ and $\tau$ on $\Pi^{c} \otimes H_{\nu}$ explicit. The theorem we shall now derive allows to decide, for any given irreducible product representation of the CCR, whether any given $f=(\underline{s}, \underline{h})$ or $g=(\underline{t}, \underline{h})$ is a good test function, i.e. whether $\underline{s} \in \sigma, \underline{t} \in \tau$, or not.

Theorem Let $\Pi_{\nu}^{c} \otimes H_{\nu}$ be any IDPS and $\chi \in \Pi_{\nu}^{c} \otimes H_{\nu}$ a $c_{0}$-vector with $\left\|\chi_{\nu}\right\|=1 \forall v$. Then $W(\lambda \underline{s})$ is a unitary operator, continuous in $\lambda$ and obeying the CCR if and only if
A) $\sum_{\nu=1}^{\infty}\left|s_{\nu}^{n}\left(\chi_{v}, q_{v}^{n} E_{\nu} \chi_{v}\right)\right|<\infty \quad n=1,2$
B) $\sum_{\nu=1}^{\infty}\left(\chi_{v},\left(1-E_{v}\right) \chi_{v}\right)<\infty$

where

$$
\left(E_{\nu} \chi_{\nu}\right)(\xi) \equiv\left\{\begin{array}{l}
\Theta\left(\frac{c}{\left|s_{v}\right|}-|\xi|\right) \chi_{\nu}(\xi) \quad s_{\nu} \neq 0 \\
\chi_{v}(\xi) s_{\nu}=0
\end{array}\right.
$$

The proof is based on a lemma following from Kolmogorov's three series theorem [17].

Lemma 7. Suppose that $\varrho_{v}\left(x_{v}\right) v=1,2, \ldots$ are probability densities with characteristic functions

$$
\varphi_{\nu}(\lambda)=\int e^{-i \lambda x_{v}} \varrho_{\nu}\left(x_{v}\right) d x_{\nu} .
$$

Let $c>0$ and $x_{\nu}^{\prime}=\Theta\left(c-\left|x_{\nu}\right|\right) x_{\nu}$ and let

$$
m_{\nu}^{\prime}=\int x_{\nu}^{\prime} \varrho_{\nu}\left(x_{v}\right) d x_{v} \quad \sigma_{\nu}^{\prime 2}=\int\left(x_{\nu}^{\prime}-m_{v}^{\prime}\right)^{2} \varrho_{\nu}\left(x_{v}\right) d x_{\nu}
$$

be the means and mean square deviations of $x_{\nu}^{\prime}$ and

$$
p_{\nu}^{\prime}=\int \Theta\left(\left|x_{\nu}\right|-c\right) \varrho_{\nu}\left(x_{\nu}\right) d x_{\nu}
$$

the probability for $\left|x_{v}\right|>c$.

Then the following two conditions are equivalent

(1) $\prod_{\nu=1}^{n} \varphi_{\nu}(\lambda)$ converges to a characteristic function

(2) $\Sigma m_{v}^{\prime}, \Sigma \sigma_{v}^{\prime 2}$ and $\Sigma p_{v}^{\prime}$ all converge.

Remark. Absolute convergence in condition (1) is equivalent to convergence after any permutation of the indices $v$, and this to absolute convergence in (2) [18].

Proof of the theorem. From Lemma 7 one deduces the equivalence of conditions $\mathrm{A}, \mathrm{B}$ with the fact that

$$
w(\lambda) \equiv \lim _{n \rightarrow \infty} \prod_{\nu=1}^{n}\left(\chi_{v}, e^{i \lambda q_{v} s_{v}} \chi_{v}\right)
$$


is a characteristic function (in $\lambda$ ) and that the convergence on the rhs. is absolute. To verify the "only if" part we note that the continuous one parameter unitary group $W(\lambda \underline{s})$ has a spectral decomposition [13]

so that

$$
W(\lambda \underline{s})=\int_{-\infty}^{+\infty} e^{i \lambda x} d E(x)
$$

$$
w(\lambda)=(\chi, W(\lambda \underline{s}) \chi)=\int_{-\infty}^{+\infty} e^{i \lambda x}(\chi, d E(x) \chi)
$$

is indeed the characteristic function of the probability measure $(\chi, d E \chi)$ (note that $\int_{-\infty}^{+\infty}(\chi, d E(x) \chi)=(\chi, \chi)=1$ ).

To show that the converse statement is true we note that

$$
\chi \sim \widetilde{W}(\lambda \underline{s}) \chi \in H
$$

follows from the existence of $w(\lambda)$.

But then $\widetilde{W}(\lambda \underline{s}) \chi \sim \psi \forall c_{0}$-vectors $\psi \in \Pi \stackrel{c(\chi)}{\otimes} H_{v}$ because of transitivity, whereas the unitarity of $\widetilde{W}(\lambda \underline{s})$ implies

i.e.

$$
\widetilde{W}(-\lambda \underline{s}) \psi \sim \chi
$$

$$
\widetilde{W}(\lambda \underline{s}) \psi \in \prod_{\nu}^{c(x)} H_{v}
$$

and

$$
\widetilde{W}^{-1}(\lambda \underline{s}) \psi=\widetilde{W}(-\lambda \underline{s}) \psi \in \prod_{\nu}^{c(x)} H_{\nu}
$$

Since the $\psi \operatorname{span} \Pi_{\nu}^{c(x)} H_{\nu}$ and $\widetilde{W}(\lambda \underline{s})$ is unitary in $H$ so that $\widetilde{W}(\lambda \underline{s}) \varphi$ is continuous in $\varphi$ we finally find that the restriction $\widetilde{W}^{c}(\lambda \underline{s}) \equiv W(\lambda \underline{s})$ is unitary in $\prod_{\nu}^{c(x)} \otimes H_{\nu}$ qed.

Corollary 1. Let $\Pi^{c} \otimes H_{\nu}$ be any IDPS. Then $W(\lambda \underline{s})$ is a unitary operator continuous in ${ }^{\nu} \lambda$ and obeying the CCR iff there is a non zero $c_{0}$-vector $\psi \in \Pi^{c} \otimes H_{\nu}$ with

$$
\sum_{\nu=1}^{\infty}\left|s_{\nu}^{n}\left(\psi_{\nu}, q_{\nu}^{n} \psi_{\nu}\right)\right|<\infty \quad n=1,2 .
$$

Proof. Under the conditions A), B) of the preceding theorem

$$
\psi=\prod_{v=1}^{\infty} \otimes E_{v} \chi_{v}
$$

is such a vector; condition B) may be written as $\psi \sim \chi$ which ensures that $\psi$ is in the IDPS of $\chi$. 
Let now be $\psi$ any vector obeying the corollary conditions, and $\left\|\psi_{\nu}\right\|=1$ without loss of generality.

Since

$$
\left|\left(\psi_{\nu}, q_{\nu}^{2} E_{\nu} \psi_{\nu}\right)\right| \leqq\left|\left(\psi_{\nu}, q_{\nu}^{2} \psi_{\nu}\right)\right|
$$

condition $A, n=2$ of the theorem holds.

$$
s_{v}^{2}\left(\psi_{v}, q_{\nu}^{2} \psi_{v}\right) \geqq c^{2}\left(\psi_{v},\left(1-E_{v}\right) \psi_{v}\right)
$$

implies condition $\mathrm{B}$. To derive $A, n=1$ we note that

$$
\begin{aligned}
\left|s_{\nu}\left(\psi_{\nu}, q_{\nu} E_{\nu} \psi_{\nu}\right)\right| & \leqq\left|s_{\nu}\left(\psi_{\nu}, q_{\nu} \psi_{\nu}\right)\right|+c^{-1}\left|s_{\nu}^{2}\left(\psi_{\nu}, q_{\nu}^{2}\left(1-E_{\nu}\right) \psi_{\nu}\right)\right| \\
& \leqq\left|s_{\nu}\left(\psi_{\nu}, q_{\nu} \psi_{\nu}\right)\right|+c^{-1}\left|s_{\nu}^{2}\left(\psi_{\nu}, q_{\nu}^{2} \psi_{\nu}\right)\right|
\end{aligned}
$$

and that the sum over $v$ of the rhs. converges.

Obviously, analogous statements result for $V(\underline{t}), \underline{t} \in \tau$ :

Corollary 2. The above theorem and corollary remain valid after the exchanges

$$
\begin{aligned}
\sigma & \rightarrow \tau \\
\underline{s} & \rightarrow \underline{t} \\
W(\underline{s}) & \rightarrow V(\underline{t}) \\
\chi_{v}(\xi) & \rightarrow \tilde{\chi}_{v}(k) \\
q_{v} & \rightarrow p_{v} \\
\xi & \rightarrow k .
\end{aligned}
$$

Finally, as an example, we shall take a short look at the IDPS generated by the equivalence class of the $c_{0}$-vector $\varphi$ with

$$
\varphi_{\nu}(\xi)=2^{-1 / 2} \Theta(|\xi|-1)|\xi|^{-1} \forall \nu .
$$

Straightforward calculations show that in this case

$$
\underline{s} \in l_{1}
$$

is equivalent to condition B of our theorem, and sufficient for condition A to be fulfilled, so that

$$
\sigma=l_{1}
$$

In connection with the first corollary the question will arise whether there is any one $c_{0}$-vector $\psi$ in the IDPS such that

$$
\sum_{\nu}\left|s_{\nu}^{n}\left(\psi_{v}, q_{\nu}^{n} \psi_{v}\right)\right|<\infty \quad n=1,2
$$

is equivalent to $\underline{s}$ being an element of $\sigma$.

The above example illustrates that this is not generally true. For if $\psi$ were such a vector

$$
\sum_{\nu} s_{\nu}^{2}\left(\psi_{\nu}, q_{\nu}^{2} \psi_{\nu}\right)<\infty \quad \forall \underline{s} \in l_{1}
$$

would imply $\left(\psi_{v}, q_{v}^{2} \psi_{v}\right)<M<\infty \forall v$. 
On the other hand for $\varphi$ and $\psi$ to be equivalent $\left(\varphi_{\nu}, \psi_{\nu}\right)$ must tend to 1 as $v \rightarrow \infty$. This yields

$$
\left(\psi_{\nu}, \Theta\left(\left|q_{\nu}\right|-c\right) \psi_{\nu}\right) \geqq(4 c)^{-1}
$$

for any $c \geqq 1$ and almost all $\nu$, so that for these $v$

$$
\left(\psi_{v}, q_{\nu}^{2} \psi_{\nu}\right) \geqq c^{2}\left(\psi_{\nu}, \Theta\left(\left|q_{v}\right|-c\right) \psi_{\nu}\right) \geqq c / 4
$$

which provides a contradiction if $c \geqq 4 M$.

We conclude that in general it will not be possible to improve the statements of our theorem and corollaries in the above mentioned manner.

Acknowledgements. This work has its origin in Professor R. Jost's lectures on Quantum Field Theory [3], where he already sketched the proof of the KLAUDER, McKenNa and Woods theorem, and in helpful discussions which are gratefully acknowledged. Further thanks are due to Professor KLaUder for kindly sending preprints of his work and for an ensuing correspondence, and to the „Deutsche Forschungsgemeinschaft" for financial support.

\section{References}

[1] Friedrichs, K. O.: Mathematical aspects of the quantum theory of fields. New York: Interscience 1953. - HAAG, R.: Math.-Fys. Medd. Danske Vid. Selsk. 29, no. 12 (1955). — SEGAL, J. E.: Lectures in applied mathematics. Vol. II. Providence: Am. Math. Soc. 1963.

[2] Glauber, R. J.: Phys. Rev. 131, 2766 (1963).

[3] Jost, R.: Einführung in die Theorie quantisierter Felder. (Unpublished lectures, Zürich 1965/66.)

[4] Chung, V.: Phys. Rev. 140B, 1110 (1965).

[5] Araki, H., and E. J. Woods: J. Math. Phys. 4, 637 (1963). — cf. also H. Araki, W. Wrss: Helv. Phys. Acta 37, 136 (1964). - Verboven, E. J.: Phys. Letters 21, 391 (1966).

[6] HAAG, R.: Nuovo Cimento 25, 287 (1962).

[7] Klauder, J. R., and J. McKenna: J. Math. Phys. 6, 68 (1965). - Klauder, J. R., J. McKenNA, and E. J. Woods: Direct product representations of the canonical commutation relations (preprint 1965).

[8] v. Neumann, J.: Compos. Math. 6, 1 (1938).

[9] - Math. Ann. 104, 570 (1931).

[10] LEw, J. S.: The structure of representations of the CCR, unpublished thesis, p. 57, Princeton 1960.

[11] Dixmier, J.: Les algèbres d'opérateurs dans l'espace hilbertien, p. 18. Paris: Gauthiers-Villars 1957.

[12] Araki, H.: J. Math. Phys. 1, 492 (1960).

[13] Riesz, F., u. B. Sz.-NAGY : Vorlesungen über Funktionsanalysis, p. 366. Berlin: Deutscher Verlag der Wissenschaften 1956.

[14] cf. e.g. Smirnow, W. L.: Lehrgang der höheren Mathematik V, p. 281. Berlin: Deutscher Verlag der Wissenschaften 1962.

[15] cf. e.g. v. Neumann, J.: Math. Ann. 102, 370 (1929).

[16] v. Neumann, J.: Ann. Math. 33, 567 (1932).

[17] cf. e.g. PITT, H. R.: Integration, measures and probability. Edinburgh: Oliver \& Boyd 1963. - Dоoв, J. L.: Stochastic processes. New York: Wiley 1953.

[18] cf. e.g. KNopr, K.: Theorie und Anwendung der unendlichen Reihen, pp. 140, 234. Berlin: Springer 1947. 